\title{
ReaR

\section{Bloqueo del plexo cervical superficial para cirugía de resección de glándula paratiroides en régimen ambulatorio. A propósito de un caso}

Eced Sánchez M, Alonso Fernández M, Tejón Pérez G, Álvarez Cebrián MA, Alfaro Martínez V, Garicano Goldaraz C.

Hospital Universitario Marqués de Valdecilla, Cantabria.

\begin{abstract}
Resumen
El bloqueo del plexo cervical superficial ecoguiado es una técnica sencilla y que, complementada con una adecuada sedación, puede proporcionar las condiciones adecuadas para la realización de la exéresis de la glándula paratiroides sin necesidad de anestesia general. Dicha técnica anestésica favorece el alta temprana de los pacientes sometidos a este tipo de cirugía, que pueden de este modo verse más fácilmente incluidos en un régimen de Cirugía Mayor Ambulatoria.

Es una técnica indicada para cirugías de corta duración, poco complejas, en pacientes colaboradores; aunque también estaría indicada en pacientes de alto riesgo evitando la necesidad de una anestesia general y asegurando una mayor estabilidad hemodinámica.
\end{abstract}

\section{Introducción}

El bloqueo del plexo cervical superficial ecoguiado es una técnica sencilla y que, complementada con una adecuada sedación, puede proporcionar las condiciones adecuadas para la realización de la exéresis de la glándula paratiroides sin necesidad de anestesia general. Dicha técnica anestésica favorece el alta temprana de los pacientes sometidos a este tipo de cirugía, que pueden de este modo verse más fácilmente incluidos en un régimen de Cirugía Mayor Ambulatoria (2).

Es una técnica indicada para cirugías de corta duración, poco complejas, en pacientes colaboradores; aunque también estaría indicada en pacientes de alto riesgo evitando la necesidad de una anestesia general y asegurando una mayor estabilidad hemodinámica $(1,3)$.
Aunque los efectos adversos son escasos, debemos conocerlos. Entre estos se encuentran: parálisis ipsilateral del nervio frénico $(80 \%)$ y del laríngeo recurrente $(50 \%)$, síndrome de Horner $(20 \%)$, parálisis del nervio facial, inyección accidental del anestésico en el espacio epidural o intradural provocando una anestesia espinal total o epidural alta y punción hemática con reabsorción vascular del anestésico $(4,5)$.

\section{Descripción del caso}

Presentamos el caso de una mujer de 35 años, sin antecedentes médicos de interés salvo asma bronquial moderado persistente en tratamiento con un agonista beta 2 de acción larga.

Fue diagnosticada de hiperparatiroidismo primario por adenoma de la glándula paratiroides superior derecha, con escasa respuesta al tratamiento médico; por lo que se 
programa para cirugía de resección del adenoma como tratamiento definitivo.

Desde la consulta de Cirugía General se le ofrece la posibilidad de realizar la intervención mediante un abordaje mínimamente invasivo y la inclusión en el régimen de CMA hospitalario. La paciente entiende las ventajas y acepta.

En la consulta de preanestesia se solicita ECG, bioquímica y hemograma, que son normales, además de contar con las cifras de calcio y PTH previas. Se constata que la paciente es colaboradora y se le explica la técnica de anestesia regional más sedación consciente que se va a utilizar durante la intervención, mostrándose en todo momento de acuerdo.

En el quirófano se administran $2 \mathrm{mg}$ de midazolam ev como ansiolítico y se procede a realizar el bloqueo cervical ecoguiado. Se utiliza sonda lineal de alta frecuencia y se comienza realizando exploración de la región cervical derecha, siguiendo el plexo braquial desde su localización junto a la arteria subclavia en la región supraclavicular hasta su situación entre los músculos escalenos. Se identifican bajo el músculo esternocleidomastoideo las raíces nerviosas correspondientes al plexo cervical superficial a la altura de C4 (Imagen 1 y 2). Se depositan a ese nivel $6 \mathrm{ml}$ de levobupivacaína al 0,5\%, observándose la correcta difusión del mismo. También se infiltran $5 \mathrm{ml}$ de levobupivacaína al $0,5 \%$ en el tejido celular subcutáneo en la línea posterior del músculo esternocleidomastoideo para ampliar al mayor número de ramas posible.

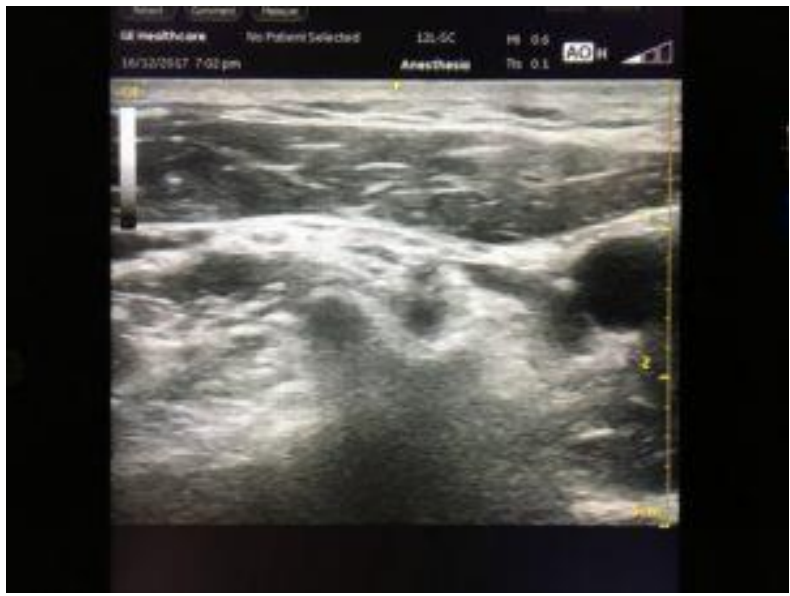

Imagen 1. Plexo cervical superficial

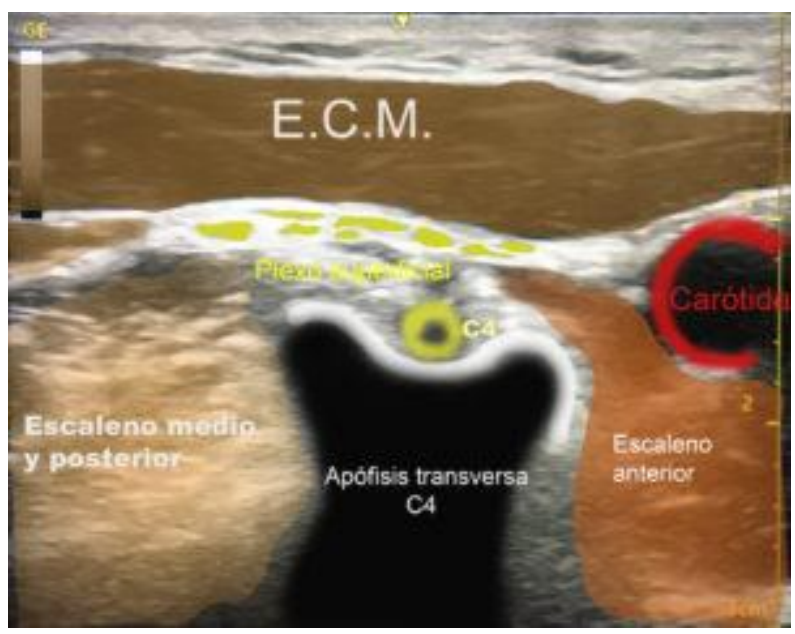

Imagen 2. Plexo cervical superficial dibujo

Una vez constatada la anestesia de la región cervical derecha, se inicia perfusión continua de Remifentanilo en régimen de TCI (Minto) con 1,5 ng/ml de diana en el efecto. La cirugía se realiza a través de una incisión paramedial derecha de $4 \mathrm{~cm}$ aproximadamente. Se identifica glándula paratiroides superior derecha, que se extirpa sin incidencias.

La paciente permaneció en todo momento colaboradora, sin requerir profundizar en la sedación ni analgesia complementaria salvo infiltración con 2 $\mathrm{ml}$ de lidocaína al $2 \%$ en fascia profunda del cuello por molestia a ese nivel. Antes de salir de quirófano se administra $1 \mathrm{~g}$ de Paracetamol y $50 \mathrm{mg}$ de Dexketoprofeno y se traslada a la URPA. 
En la URPA permanece durante una hora con buen control del dolor sin requerir rescates de opioides y $\sin$ presentar náuseas ni vómitos. Evoluciona favorablemente sin ningún tipo de complicaciones siendo dada de alta hospitalaria el mismo día.

\section{Discusión}

El bloqueo del plexo cervical superficial ha sido ampliamente utilizado en la endarterectomía carotídea y en discectomía cervical anterior; al permitir mayor estabilidad hemodinámica así como mejor mantenimiento de la perfusión cerebral, al permanecer el paciente despierto (1). Por tanto, se propone como una técnica segura que ofrece ventajas en la cirugía de cuello.

Así, con este caso que presentamos, se observa la posibilidad de realizar paratiroidectomías mínimamente invasivas bajo técnicas de anestesia locorregional ecoguiadas como es el bloqueo del plexo cervical superficial acompañadas de una adecuada sedación (el disconfort del paciente es una causa de reconversión de anestesia regional a general).

En nuestro caso, la paciente permaneció confortable gracias a la realización del bloqueo y la sedación acompañante. Esta técnica se asoció a menor dolor postoperatorio y menor incidencia de náuseas y vómitos, sin requerir dosis de opioides de rescate ni fármacos antieméticos (3). Todo esto, asociado a la cirugía mínimamente invasiva, favoreció la buena evolución de la paciente y el alta hospitalaria temprana en régimen ambulatorio.

\section{Conclusiones}

El bloqueo del plexo cervical superficial ecoguiado para parotiroidectomía mínimamente invasiva es una buena alternativa a la anestesia general en pacientes seleccionados, con ciertas ventajas:

- $\quad$ Permite una anestesia y analgesia intraoperatorias adecuadas.

- Se asocia a menor dolor postoperatorio, náuseas y vómitos.

- Menor coste hospitalario, al precisar menor dosis de opioides y de fármacos antieméticos, menos días de ingreso y menor consumo de recursos.

- Técnica segura con pocos efectos secundarios.

- Permite valorar la afectación del nervio laríngeo recurrente al estar el paciente despierto.

- Posibilidad de realizar este tipo de cirugías en régimen de CMA, al poder dar de alta a dichos pacientes en el día.

\section{Bibliografía}

1. Wang H, Ma L, Yang D, Wang T, Wang Q, Zhang L. Cervical plexus anesthesia versus general anesthesia for anterior cervical discectomy and fusion surgery: A randomized clinical trial. Medicine. 2017; 96:7. (PubMed) (HTML) (PDF)

2. Pintaric T, Hocevar M, Jereb S, Casati A, Novak Jankovic V. Prospective, Randomized Comparison Between Combined (Deep and Superficial) and Superficial Cervical Plexus Block with Levobupivacaine for Minimally Invasive Parathyroidectomy. ( $\underline{\text { PubMed }}$ )

3. Suri KB, Hunter CW, Davidov T, Anderson MB, Dombrovskiy VY, Trooskin SZ. Postoperative recovery advantages in patients undergoing thyroid and parathyroid surgery under regional anesthesia. UMDNJ-Robert Wood Johnson Medical School, New Brunswick, NJ, USA. (HTML)

4. Ortega Romero A, De Diego Isasa D, Del Olmo Rodríguez C Maroto Ramos E, Rouco Gil R. Ecografía portátil en anestesia regional: bloqueos del plexo braquial. Departamento de 
Anestesiología. Hospital ASEPEYO. Coslada, Madrid. (PDF)

5. Gil S, Pretel M, Madrazo M, Redondo J, Sarabia R, Bernal G. Bloqueo del plexo cervical en paratiroidectomía mínimamente invasiva. A propósito de tres casos. Departamento de Anestesiología y Reanimación. Hospital General de Ciudad Real. (PDF)
Manuel Eced Sánchez manueleced@gmail.com MIR Anestesiología. Hospital Universitario Marqués de Valdecilla, Cantabria.

Aceptado para blog en abril de 2018.

Correspondencia al autor 